\title{
Feature Extraction and Classification Methods for a Motor Task Brain Computer Interface: A Comparative Evaluation for Two Databases
}

\author{
Oana Diana Eva \\ Faculty of Electronics, Telecommunications and \\ Information Technology \\ "Gheorghe Asachi” Technical University \\ Institute of Computer Science \\ Romanian Academy \\ Iasi, Romania
}

\author{
Anca Mihaela Lazar \\ Faculty of Medical Bioengineering \\ "Grigore T. Popa" University of Medicine and Pharmacy \\ Iasi, Romania
}

\begin{abstract}
A comparative evaluation is performed on two databases using three feature extraction techniques and five classification methods for a motor imagery paradigm based on Mu rhythm. In order to extract the features from electroencephalographic signals, three methods are proposed: independent component analysis, Itakura distance and phase synchronization. The last one consists of: phase locking value, phase lag index and weighted phase lag index. The classification of the extracted features is performed using linear discriminant analysis, quadratic discriminant analysis, Mahalanobis distance based on classifier, the k-nearest neighbors and support vector machine. The aim of this comparison is to evaluate which feature extraction method and which classifier is more appropriate in a motor brain computer interface paradigm. The results suggest that the effectiveness of the feature extraction method depends on the classification method used.
\end{abstract}

Keywords-Brain computer interface; independent component analysis; Itakura distance; phase synchronization; classifiers

\section{INTRODUCTION}

Brain Computer Interface (BCI) provides a new communication method for people who are suffering of motor disabilities [1]. A BCI system acquires brain signals, analyzes them and translates them into commands for external devices (wheelchair, neuroprosthesis, etc.). The most commonly studied signals generated from brain activity are electrical signals. The electroencephalography (EEG) records the electrical activity by using electrodes placed on the scalp.

Motor imagery produces reliable and distinct features in the brain activity that can be used by BCI systems. When a user performs a mental activity as left/right hand movement imagination without physically executing the movements, changes called event related desynchronizations (ERD) and event related synchronizations (ERS) appear in the sensorimotor area in the corresponding signal power of $\mathrm{Mu}$ or beta rhythms. Mu rhythm represents an oscillation of the EEG signal in the frequency band $8-12 \mathrm{~Hz}$ and it is affected by movements and movement imagery [2]. There are different features extraction methods for EEG signals suited to discriminate the motor tasks in a BCI paradigm. Among these, the independent component analysis [3], [4], Itakura distances
[5]-[7] and phase synchronization methods [8]-[10] are chosen in order to be used for classification with linear discriminant analysis [11], quadratic discriminant analysis [12], Mahalanobis distance [13], the k-nearest neighbors [14], [15] and support vector machine [16], [17].

In Section II there are described the databases used in the comparative study. Section III is reserved to the methods used in the proposed assessing. The results obtained for the used databases are presented in Section IV and Section V contains the conclusions of the paper.

\section{DATABASES}

In the evaluation of efficiency of feature extraction and classification methods, two databases are used: the database composed of EEG signals recorded in our laboratory and the BCI competition 2002 database downloaded from the internet [18]. The databases description is listed in Table 1.

TABLE I. DATABASES DESCRIPTION

\begin{tabular}{|l|l|l|}
\hline Database details & Our database & BCI Competition 2002 \\
\hline Number of subjects & 40 & 9 \\
\hline \multirow{4}{*}{ Aquisition system } & $\begin{array}{l}\text { gMobilab+ module } \\
\text { and BCI } 2000 \\
\text { platform }\end{array}$ & Unknown \\
\hline \multirow{3}{*}{ Paradigm description } & $\begin{array}{l}\text { Left and right arrows are displayed successively on } \\
\text { a monitor. The subjects must carefully look at the } \\
\text { arrows and try to imagine the left or right hand } \\
\text { movement indicated by the arrow. }\end{array}$ \\
\hline Used channels & $\begin{array}{l}\mathrm{CP}_{3}, \mathrm{CP}_{4}, \mathrm{P}_{3}, \mathrm{C}_{3}, \\
\mathrm{Pz}_{2} \mathrm{C}_{4}, \mathrm{P}_{4}, \mathrm{Cz} .\end{array}$ & $\begin{array}{l}\mathrm{FC}_{1}, \mathrm{FC}_{2}, \mathrm{FC}_{3}, \mathrm{FC}_{4}, \mathrm{C}_{1}, \mathrm{C}_{2}, \\
\mathrm{C}_{3}, \mathrm{C}_{4}, \mathrm{CP}_{1}, \mathrm{CP}_{2}, \mathrm{CP}_{3}, \mathrm{CP}_{4}\end{array}$ \\
\hline
\end{tabular}

\section{METHODS}

The chosen feature extraction methods are presented for short. For detail information, the mentioned references may be studied.

Independent component analysis is used for spatial filters substitution. The proposed method consists in using the same spatial filter obtained by applying ICA method for relaxation state and for imagining motor tasks [19]. 
The Itakura distance for imagination of the left hand and the relaxation (rest) state is as follows [7]:

$$
I D_{R E S T-L E F T}=\log \left(\frac{M S E_{y_{R E S T}, y_{L E F T}}}{M S E_{y_{R E S T}, y_{R E S T}}}\right),
$$

where the mean square error $M S E_{y_{R E S T}, y_{L E F T}}$ and $M S E_{y_{R E S T} y_{R E S T}}$ are:

$$
\begin{aligned}
& M S E_{y_{R E S T}, y_{L E F T}}=\left(a^{L E F T}\right)^{T} R_{y_{R E S T}}(p) a^{L E F T}, \\
& M S E_{y_{R E S T} y_{R E S T}}=\left(a^{R E S T}\right)^{T} R_{y_{R E S T}}(p) a^{R E S T}
\end{aligned}
$$

and $R_{y_{R E S T}}(p)$ is the autocorrelation matrix of $y_{R E S T}(n)$, $y_{R E S T}(n)$ is the output of an autoregressive (AR) model system with an input of $x_{R E S T}(n)$.

The autoregressive model is characterized by:

$$
y(n)=-\sum_{k=1}^{p} a_{k} y(n-k)+e(n),
$$

$a_{k}$ are the parameters of the model, $p$, the model order and $e(n)$ the prediction error.

There are similar relations for the Itakura distance for movement imagination of the right hand and the relaxation state.

The left symmetric Itakura distance is [20]:

$$
I D_{L E F T}=\frac{1}{2}\left(I D_{R E S T-L E F T}+I D_{L E F T-R E S T}\right) .
$$

The left normalized Itakura distance is defined as [21]:

$$
N O R M_{I D_{R E S T-L E F T}}=\frac{\left(I D_{R E S T-L E F T}-\min \left(I D_{R E S T-L E F T}\right)\right) * 100}{\max \left(I D_{R E S T-L E F T}\right)-\min \left(I D_{R E S T-L E F T}\right)} .
$$

Phase locking value (PLV) [22], phase lag index (PLI) [23] and weighted phase lag index (wPLI) [24] are used to measure the synchronization between two signals $x(t)$ and $y(t)$.

PLV characterizes the stability of the phase difference between instantaneous phases $\varphi_{x}(t)$ and $\varphi_{y}(t)$ :

$$
\begin{aligned}
& P L V=\left|\left\langle e^{j \Delta \varphi(t)}\right\rangle\right| \\
& \quad \Delta \varphi(t)=\varphi_{y}(t)-\varphi_{x}(t) .
\end{aligned}
$$

The phase lag index [23] is defined by:

$$
P L I=\left|\left\langle\operatorname{sign}\left[\Delta \theta\left(t_{k}\right)\right]\right\rangle\right|,
$$

sign is the signum function and $<$. $>$ denotes the average over the time.

The weighted phase lag index is calculated using [11]:

$$
w P L I=|\langle I(X)\rangle| /\langle|I(X)|\rangle=|\langle I|X| \operatorname{sign} I(X)\rangle| /\langle|I(X)|,
$$

where $I(X)$ is the imaginary component of the cross spectrum between two signals $x(t)$ and $y(t)$.

The used methods are described in detail in [24]-[26].

\section{RESULTS}

In this section there are presented both comparisons between some features extraction methods and comparisons between some classification methods used for EEG signals recorded in a BCI motor task paradigm. The results are reported on two EEG databases: the 2002 BCI Competition database and our own database.

\section{A. Database of EEG Signals Recorded in Our Laboratory}

The methods used in feature extraction used for our database are: independent component analysis, Itakura distance, symmetric Itakura distance and measures for phase synchronization. For ICA three algorithms (INFOMAX, SOBI and JADE) are used. Concerning Itakura distance and symmetric Itakura distance, 6 and 10 order AR models are handled. PLV, PLI and wPLI are applied measures for phase synchronization. LDA, QDA, MD, kNN ( $\mathrm{k}=1: 5)$ and SVM are the methods we have utilized in order to classify the detected features.

In Table 2, the mean and maximum correct classification rates acquired for each of the mentioned feature extraction methods are presented. For ICA, Itakura distance and

\begin{tabular}{|c|c|c|c|c|c|c|c|}
\hline \multirow{3}{*}{\multicolumn{2}{|c|}{ Method }} & \multicolumn{6}{|l|}{ Classification rates } \\
\hline & & \multicolumn{2}{|l|}{ LDA } & \multicolumn{2}{|l|}{ QDA } & \multicolumn{2}{|l|}{ MD } \\
\hline & & $\begin{array}{l}\text { Mean } \\
\pm \\
\text { standard deviation }[\%]\end{array}$ & $\operatorname{Max}[\%]$ & $\begin{array}{l}\text { Mean } \\
\pm \\
\text { standard deviation }[\%]\end{array}$ & $\begin{array}{l}\operatorname{Max} \\
{[\%]}\end{array}$ & $\begin{array}{l}\text { Mean } \\
\pm \\
\text { standard deviation }[\%]\end{array}$ & $\begin{array}{l}\text { Max } \\
{[\%]}\end{array}$ \\
\hline \multirow{3}{*}{ ICA } & INFOMAX & $81,3 \pm 12,74$ & 97,73 & $83,6 \pm 15,9$ & 100 & $82,28 \pm 15,82$ & 100 \\
\hline & SOBI & $78,8 \pm 15,63$ & 97,78 & $79,3 \pm 17,66$ & 100 & $79,64 \pm 17,52$ & 100 \\
\hline & JADE & $83,90 \pm 12,39$ & 100 & $82,61 \pm 19,52$ & 100 & $83,62 \pm 15,59$ & 100 \\
\hline \multirow{2}{*}{$\begin{array}{l}\text { Itakura } \\
\text { Distance }\end{array}$} & Model Order 6 & $82,40 \pm 12,60$ & 100 & $88,19 \pm 9,74$ & 100 & $86,62 \pm 11,28$ & 100 \\
\hline & Model Order 10 & $83,35 \% \pm 11,94$ & 100 & $88,33 \pm 10,22$ & 100 & $86,62 \pm 9,78$ & 98,33 \\
\hline \multirow{2}{*}{$\begin{array}{l}\text { Symmetric } \\
\text { Itakura Distance } \\
\end{array}$} & Model Order 6 & $81,35 \% \pm 15,25$ & 100 & $87,85 \pm 12,48$ & 100 & $86,75 \pm 12,35$ & 98,33 \\
\hline & Model Order 10 & $84,04 \pm 12,54$ & 100 & $89,43 \pm 10,03$ & 100 & $87,15 \pm 10,23$ & 100 \\
\hline \multirow{3}{*}{ Phase synchronization } & PLI & $64,78 \pm 7,09$ & 82,12 & $73,98 \pm 6,64$ & 85,28 & $73,08 \pm 6,35$ & 84,67 \\
\hline & PLV & $64,62 \pm 7,18$ & 82,48 & $73,99 \pm 6,67$ & 85,64 & $73,03 \pm 6,51$ & 84,31 \\
\hline & wPLI & $59,06 \pm 3,62$ & 66,67 & $64,08 \pm 4,67$ & 72,51 & $63,06 \pm 4,44$ & 71,78 \\
\hline
\end{tabular}
symmetric Itakura distance methods, maximum classification rates were obtained for LDA, QDA and MD. The lowest classification rates were achieved for PLI, PLV and wPLI. The mean classification rates are in the range of $59.06 \%$ (for wPLI) and $89.43 \%$ (for symmetric Itakura distance). The highest mean and maximum values of the classification rates were obtained using QDA.

TABLE II. THE MEAN AND MAXIMUM ClASSIFICATION RATES FOR ICA, ITAKURA DistANCE, SYMMETRIC ITAKURA DistaNCE AND PHASE SYNCHRONIZATION METHODS WITH LDA, QDA AND MD CLASSIFIERS (ON OUR DATABASE) 
From the analysis of data in Table 2, the outcomes are as follows:

- For ICA method, JADE algorithm performs the best classification rates for LDA and MD classifiers.

- For Itakura distance and symmetric Itakura distance methods, 10 order AR model with QDA classifier presents the best performance.

- For PLI, PLV and wPLI, QDA classifier attends the highest classification rates.

In Table 3, the mean and maximum correct classification rates obtained for the each of the mentioned methods with kNN classifier are presented.

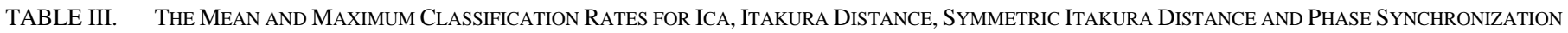
METHODS WITH KNN CLASSIFIER (ON OUR DATABASE)

\begin{tabular}{|c|c|c|c|c|}
\hline & & kNN & Classification rates & \\
\hline Method & & & $\begin{array}{l}\text { Mean } \\
\pm\end{array}$ & Max \\
\hline & & 1 & $81,76 \pm 13,77$ & 100 \\
\hline & & 2 & $81,76 \pm 13,76$ & 100 \\
\hline & INFOMAX & 3 & $81,79 \pm 13,75$ & 100 \\
\hline & & 4 & $81,80 \pm 13,74$ & 100 \\
\hline & & 5 & $81,83 \pm 13,74$ & 100 \\
\hline & & 1 & $82,25 \pm 13,78$ & 100 \\
\hline & & 2 & $82,21 \pm 13,79$ & 100 \\
\hline ICA & SOBI & 3 & $82,17 \pm 13,82$ & 100 \\
\hline & & 4 & $82,14 \pm 13,84$ & 100 \\
\hline & & 5 & $82,11 \pm 13,87$ & 100 \\
\hline & & 1 & $84,61 \pm 13,81$ & 99,80 \\
\hline & & 2 & $84,61 \pm 13,81$ & 99,80 \\
\hline & JADE & 3 & $84,62 \pm 13,80$ & 99,80 \\
\hline & & 4 & $84,62 \pm 13,79$ & 99,80 \\
\hline & & 5 & $84,63 \pm 13,78$ & 99,81 \\
\hline & & 1 & $84,69 \pm 9,92$ & 97,50 \\
\hline & & 2 & $84,04 \pm 9,90$ & 97,78 \\
\hline & Model order 6 & 3 & $83,56 \pm 9,49$ & 97,08 \\
\hline & & 4 & $83,34 \pm 9,50$ & 97,00 \\
\hline Itakura & & 5 & $82,58 \pm 9,62$ & 96,94 \\
\hline Distance & & 1 & $85,00 \pm 9,91$ & 97,50 \\
\hline & & 2 & $84,46 \pm 10,18$ & 97,22 \\
\hline & Model order 10 & 3 & $84,12 \pm 10,33$ & 97,08 \\
\hline & & 4 & $83,93 \pm 10,56$ & 97,00 \\
\hline & & 5 & $83,40 \pm 10,68$ & 96,94 \\
\hline & & 1 & $84,55 \pm 11,54$ & 99,17 \\
\hline & & 2 & $83,81 \pm 11,61$ & 99,44 \\
\hline & Model order 6 & 3 & $83,50 \pm 11,52$ & 99,17 \\
\hline & & 4 & $83,43 \pm 11,56$ & 99,33 \\
\hline $\begin{array}{l}\text { Symmetric } \\
\text { Itakura }\end{array}$ & & 5 & $82,93 \pm 11,50$ & 98,61 \\
\hline $\begin{array}{l}\text { Itakura } \\
\text { Distance }\end{array}$ & & 1 & $86,10 \pm 16,96$ & 99,17 \\
\hline & & 2 & $85,71 \pm 17,06$ & 99,44 \\
\hline & Model order 10 & 3 & $85,00 \pm 16,89$ & 98,75 \\
\hline & & 4 & $84,80 \pm 16,91$ & 98,67 \\
\hline & & 5 & $84,22 \pm 16,85$ & 97,78 \\
\hline & & 1 & $92,74 \pm 3,42$ & 96,66 \\
\hline & & 2 & $92,83 \pm 3,40$ & 96,71 \\
\hline & PLI & 3 & $92,89 \pm 3,39$ & 96,75 \\
\hline & & 4 & $92,97 \pm 3,38$ & 96,80 \\
\hline & & 5 & $92,98 \pm 3,38$ & 96,82 \\
\hline & & 1 & $92,73 \pm 3,41$ & 96,57 \\
\hline & & 2 & $92,83 \pm 3,38$ & 96,63 \\
\hline Phase & PLV & 3 & $92,89 \pm 3,38$ & 96,67 \\
\hline & & 4 & $92,97 \pm 3,36$ & 96,70 \\
\hline & & 5 & $92,99 \pm 3,37$ & 96,72 \\
\hline & & 1 & $83,15 \pm 6,83$ & 92,94 \\
\hline & & 2 & $83,27 \pm 6,87$ & 93,06 \\
\hline & wPLI & 3 & $83,33 \pm 6,87$ & 93,16 \\
\hline & & 4 & $83,41 \pm 6,91$ & 93,28 \\
\hline & & 5 & $83,42 \pm 6,90$ & 93,33 \\
\hline
\end{tabular}


From the analysis of data in Table 3, the findings are as follows:

- For ICA method, JADE algorithm performs the best classification rates.

- For Itakura distance and symmetric Itakura distance methods, 10 order AR model offers the best performance.

- For PLI, PLV and wPLI, there are not essential differences between the classification rates.

The mean and maximum correct classification rates obtained for each of the mentioned methods with SVM classifier are organized in Table 4.

TABLE IV. THE MEAN AND MAXIMUM CLASSIFICATION RATES FOR ICA, ITAKURA DISTANCE, SYMMETRIC ITAKURA DISTANCE AND PHASE SYNCHRONIZATION METHODS WITH SVM CLASSIFIER (ON OUR DATABASE)

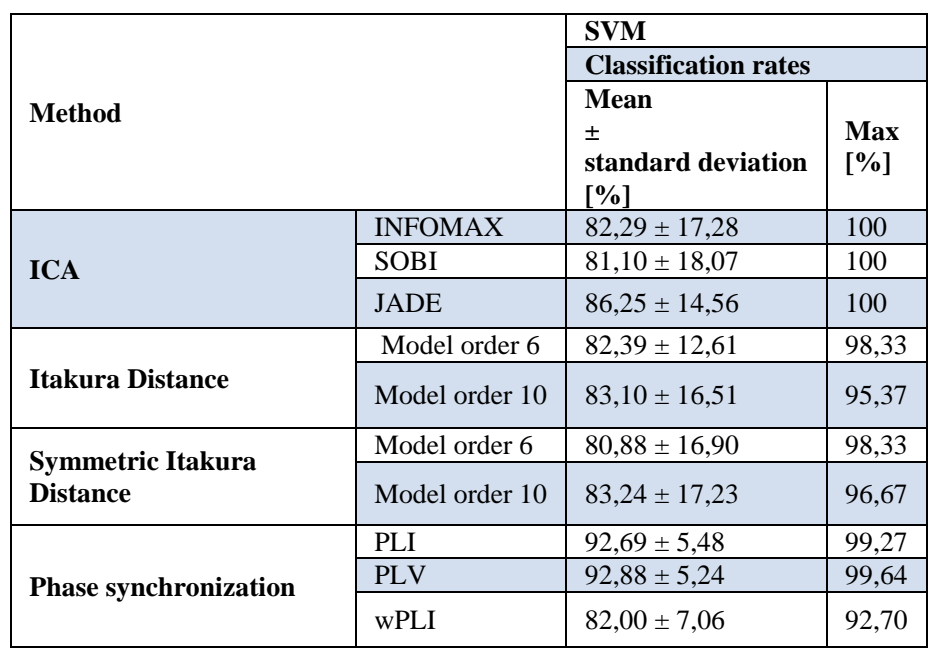

From the analysis of data in Table 4, we can conclude that:

- For ICA method, JADE algorithm performs both the highest maximum classification rate and highest mean classification rate.

- For Itakura distance and symmetric Itakura distance methods, 10 order AR model offers the best performance.

- For PLV offers the best classification rates.

\section{B. BCI Competition 2002 Database}

The methods of features extraction are the same as those for our database, except the normalized Itakura distance instead of Itakura distance and symmetric Itakura distance. It was chosen to test the method based on the normalized Itakura distance because the results obtained following the Itakura distance calculation method without the normalization procedure did not offer optimal classification rates.

The same classification methods as in the case of our database were applied.

The mean and maximum classification rates obtained with LDA, QDA and MD, $\mathrm{kNN}(\mathrm{k}=1: 5), \mathrm{SVM}$ classifiers are illustrated in Tables 5, 6 and 7, respectively.

Concerning the mean classification rates, from Table 5, we conclude that:

- For ICA, SOBI algorithm with LDA, QDA and MD classifiers lead to the best results.

- For normalized Itakura distance, 10 order AR model with LDA, QDA and MD classifier performed the best classification rates.

- For all the phase synchronization methods the highest classification rates were performed with MD classifier.

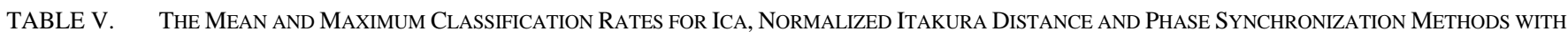
LDA, QDA AND MD CLASSIFIERS (ON BCI COMPETITION 2002 DATABASE)

\begin{tabular}{|c|c|c|c|c|c|c|c|}
\hline \multirow{3}{*}{\multicolumn{2}{|c|}{ Method }} & \multicolumn{6}{|l|}{ Classification rates } \\
\hline & & \multicolumn{2}{|l|}{ LDA } & \multicolumn{2}{|l|}{ QDA } & \multicolumn{2}{|l|}{ MD } \\
\hline & & $\begin{array}{l}\text { Mean } \\
\pm \\
\text { standard deviation }[\%]\end{array}$ & $\begin{array}{l}\operatorname{Max} \\
{[\%]}\end{array}$ & $\begin{array}{l}\text { Mean } \\
\pm \\
\text { standard deviation [\%] }\end{array}$ & $\begin{array}{l}\operatorname{Max} \\
{[\%]}\end{array}$ & $\begin{array}{l}\text { Mean } \\
\pm \\
\text { standard deviation [\%] }\end{array}$ & $\begin{array}{l}\operatorname{Max} \\
{[\%]}\end{array}$ \\
\hline \multirow{3}{*}{ ICA } & INFOMAX & $81,64 \pm 13,04$ & 97,56 & $85,62 \pm 16,99$ & 100 & $81,81 \pm 18,17$ & 100 \\
\hline & SOBI & $98,80 \pm 10,91$ & 100 & $94,10 \pm 10,16$ & 100 & $92,08 \pm 11,32$ & 100 \\
\hline & JADE & $79,91 \pm 16,88$ & 100 & $86,54 \pm 16,52$ & 100 & $83,83 \pm 14,30$ & 96,96 \\
\hline \multirow{2}{*}{$\begin{array}{l}\text { Normalized } \\
\text { Itakura Distance }\end{array}$} & Model Order 6 & $76,67 \pm 8,38$ & 82,82 & $72,86 \pm 7,87$ & 80 & $74,92 \pm 9,02$ & 83,33 \\
\hline & Model Order 10 & $80,99 \pm 7,76$ & 91,11 & $78,89 \pm 8,44$ & 86,67 & $79,63 \pm 6,16$ & 88,89 \\
\hline \multirow{3}{*}{ Phase synchronization } & PLI & $74,01 \pm 8,18$ & 86,42 & $82,24 \pm 7,07$ & 93,21 & $98,83 \pm 1,32$ & 100 \\
\hline & PLV & $74,07 \pm 8,20$ & 86,42 & $82,92 \pm 7,31$ & 93,21 & $98,49 \pm 1,34$ & 100 \\
\hline & wPLI & $76,61 \pm 6,37$ & 85,80 & $77,85 \pm 6,14$ & 88,27 & $95,88 \pm 3,72$ & 99,38 \\
\hline
\end{tabular}

The best classification rates for $\mathrm{kNN}$ classifier (Table 6) are the following:

- For ICA method, SOBI algorithm.

- For normalized Itakura distance, 10 order AR model.

- For phrase synchronization methods, PLV and PLI.
Looking at the results from Table 7, for SVM classifier, the best classification rates are the following:

- SOBI algorithm for ICA method.

- The AR model with order 10 for normalized Itakura distance method.

- PLV index for phase synchronization methods. 


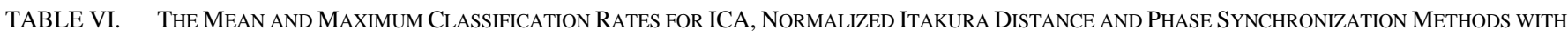
KNN CLASSIFIER (ON BCI COMPETITION 2002 DATABASE)

\begin{tabular}{|c|c|c|c|c|}
\hline & & kNN & Class & \\
\hline & & Number of neighbors & $\begin{array}{c}\text { Mean } \\
\pm\end{array}$ & Max \\
\hline & & 1 & $81,69 \pm 19,01$ & 100 \\
\hline & & 2 & $81,69 \pm 19,03$ & 100 \\
\hline & INFOMAX & 3 & $81,70 \pm 19,03$ & 100 \\
\hline & & 4 & $81,70 \pm 19,06$ & 100 \\
\hline & & 5 & $81,70 \pm 19,08$ & 100 \\
\hline & & 1 & $87,02 \pm 13,03$ & 100 \\
\hline & & 2 & $87,07 \pm 12,99$ & 100 \\
\hline ICA & SOBI & 3 & $87,12 \pm 12,94$ & 100 \\
\hline & & 4 & $87,16 \pm 12,90$ & 100 \\
\hline & & 5 & $87,20 \pm 12,86$ & 100 \\
\hline & & 1 & $79,26 \pm 17,71$ & 95,99 \\
\hline & & 2 & $79,31 \pm 17,75$ & 96,03 \\
\hline & JADE & 3 & $79,36 \pm 17,81$ & 95,96 \\
\hline & & 4 & $79,42 \pm 17,82$ & 95,88 \\
\hline & & 5 & $79,48 \pm 17,83$ & 95,81 \\
\hline & & 1 & $68,89 \pm 14,17$ & 86,67 \\
\hline & & 2 & $66,67 \pm 15,50$ & 82,22 \\
\hline & Model order 6 & 3 & $70,79 \pm 16,89$ & 86,67 \\
\hline & & 4 & $71,11 \pm 16,77$ & 88,89 \\
\hline Normalized & & 5 & $73,02 \pm 16,40$ & 88,89 \\
\hline $\begin{array}{l}\text { Itakura } \\
\text { Distance }\end{array}$ & & 1 & $72,59 \pm 10,36$ & 84,44 \\
\hline & & 2 & $71,85 \pm 11,91$ & 82,22 \\
\hline & Model order 10 & 3 & $73,33 \pm 9,16$ & 82,22 \\
\hline & & 4 & $72,84 \pm 9,01$ & 80 \\
\hline & & 5 & $75,56 \pm 9,55$ & 86,67 \\
\hline & & 1 & $99,06 \pm 0,87$ & 99,89 \\
\hline & & 2 & $99,06 \pm 0,86$ & 99,89 \\
\hline & PLI & 3 & $99,05 \pm 0,84$ & 99,89 \\
\hline & & 4 & $99,06 \pm 0,83$ & 99,90 \\
\hline & & 5 & $99,05 \pm 0,83$ & 99,90 \\
\hline & & 1 & $99,01 \pm 0,88$ & 99,89 \\
\hline & & 2 & $99,03 \pm 0,86$ & 99,89 \\
\hline $\begin{array}{l}\text { Phase } \\
\text { synchroniztion }\end{array}$ & PLV & 3 & $99,04 \pm 0,85$ & 99,89 \\
\hline & & 4 & $99,06 \pm 0,83$ & 99,90 \\
\hline & & 5 & $99,04 \pm 0,83$ & 99,90 \\
\hline & & 1 & $97,34 \pm 1,33$ & 98,46 \\
\hline & & 2 & $97,35 \pm 1,33$ & 98,48 \\
\hline & wPLI & 3 & $97,32 \pm 1,30$ & 98,51 \\
\hline & & 4 & $97,29 \pm 1,28$ & 98,54 \\
\hline & & 5 & $97,21 \pm 1,27$ & 98,56 \\
\hline
\end{tabular}

TABLE VII. MEAN AND MAXIMUM ClASSIFICATION RATES FOR ICA, NORMALIZED ITAKURA DisTANCE AND PHASE SYNCHRONIZATION METHODS WITH SVM ClASSIFIER (ON BCI COMPETITION 2002 DATABASE)

\begin{tabular}{|c|c|c|c|}
\hline \multirow[b]{3}{*}{ Method } & & \multicolumn{2}{|l|}{ SVM } \\
\hline & & \multicolumn{2}{|l|}{ Classification rates } \\
\hline & & $\begin{array}{l}\text { Mean } \\
\pm \\
\text { standard deviation }[\%]\end{array}$ & $\begin{array}{l}\text { Max } \\
{[\%]}\end{array}$ \\
\hline \multirow{3}{*}{ ICA } & INFOMAX & $82,27 \pm 15,88$ & 100 \\
\hline & SOBI & $92,59 \pm 12,16$ & 100 \\
\hline & JADE & $84,65 \pm 15,70$ & 100 \\
\hline \multirow{2}{*}{$\begin{array}{l}\text { Normalized } \\
\text { Itakura Distance }\end{array}$} & Model order 6 & $74,29 \pm 11,02$ & 84,44 \\
\hline & Model order 10 & $75,56 \pm 12,01$ & 88,89 \\
\hline \multirow{3}{*}{ Phase synchronization } & PLI & $98,56 \pm 1,06$ & 100 \\
\hline & PLV & $98,63 \pm 1,22$ & 100 \\
\hline & wPLI & $96,91 \pm 1,15$ & 98,77 \\
\hline
\end{tabular}


In order to compare our results to related works, some impediments appear. The major one is related to our dataset. As our database is not publically one, there are not reported any other results using the EEG recordings from this database. The results obtained on BCI 2002 competition dataset are consistent with other works. In [27] where a time-frequency approach is investigated are reported smaller classification rates than the classification rates obtained with methods presented. As concerning the BCI competition dataset, comparing different algorithms at present is still difficult, but as in [28] a global remark could be settled that the best choice of the classifier for a motor task BCI depends on the feature extraction method used in that system.

\section{CONCLUSIONS}

The research evaluated three feature extraction methods and five classification methods on two different databases. The algorithms are simply to apply and can be exploited by the motor imagery paradigms.

In order to have a proper preparation, the subjects from our database executed first the hand movements and then the hand movement imagination. For the subjects from the BCI competition 2002 database it is mentioned that they were well trained.

Overall the highest classification rates are obtained with QDA and with kNN classifier.

The best feature extraction methods are the phase synchronization, Itakura distance and ICA.

The results point out that the effectiveness of the feature extraction method depends on the classification method used and there is not a best method that outperforms all the others.

The future work implies the developing of a new database which will contain EEG signals achieved from people with disabilities and testing the proposed methods on that database.

\section{ACKNOWLEDGMENT}

This work was supported by a grant of the Romanian National Authority for Scientific Research and Innovation, CNCS - UEFISCDI, project number PN-II-RU-TE-2014-40832 "Medical signal processing methods based on compressed sensing; applications and their implementation".

\section{REFERENCES}

[1] J. R. Wolpaw, "Brain-computer interfaces as new brain output pathways", The Journal of Physiology, 2007, 579.3: 613-619, 2007.

[2] J. R. Wolpaw, E. W. Wolpaw, "Brain-computer interfaces: principles and practice", OUP USA, 2012.

[3] A. Kachenoura, L. Albera, L. Senhadji, P. Comon, "ICA: a potential tool for BCI systems", IEEE Signal Processing Magazine, 25(1), 57-68, 2008.

[4] A. Hyvärinen A. "Independent component analysis: recent advances", Phil Trans R Soc A 371: 20110534, http://dx.doi.org/10.1098/rsta.2011.0534, 2013.

[5] F. Ebrahimi, M. Mikaili, E. Estrada, H. Nazeran, "Assessment of Itakura distance as a valuable feature for computer-aided classification of sleep stages", In Engineering in Medicine and Biology Society, pp. 3300-3303, IEEE, 2007.

[6] Benesty J., Sondhi M. M., Huang Y., Springer Handbook of Speech Processing, Springer Science \& Business Media, 2007
[7] E. Cardoso et al., "A contribution for the automatic sleep classification based on the Itakura-Saito spectral distance", Emerging Trends in Technological Innovation, 374-381, 2010.

[8] C.J. Stam, G. Nolte, A. Daffertshofer, "Phase lag index: assessment of functional connectivity from multi channel EEG and MEG with diminished bias from common sources", Hum. Brain Mapp., vol. 28, pp. 1178-1193, 2007.

[9] X. Bao, J. Hu, "Phase synchronization for classification of motor imagery EEG”, Journal Of Information \&Computational Science, 5(2), 949-955, 2008.

[10] M. Vinck, R. Oostenveld et al., "An improved index of phasesynchronization for electrophysiological data in the presence of volumeconduction, noise and sample-size bias", Neuroimage, vol. 55(4), pp. 1548-1565, 2011.

[11] B. Blankertz, S. Lemm, M. Treder, S. Haufe, K.R. Müller, "Single-trial analysis and classification of ERP components - a tutorial", NeuroImage, 56(2), 814-825, 2011.

[12] F. Lotte, M. Congedo, A. Lécuyer, F. Lamarche, B. Arnaldi, "A review of classification algorithms for EEG-based brain-computer interfaces" J. Neural Eng., 4, pp. R1-R13, 2007.

[13] A. Bashashati, M. Fatourechi, R.K. Ward, G.E. Birch, "A survey of signal processing algorithms in brain-computer interfaces based on electrical brain signals", Journal of Neural engineering, 4(2), R32, 2007.

[14] S. Ge, R. Wang, D. Yu, "Classification of four-class motor imagery employing single-channel electroencephalography", PloS one, 9(6), e98019, 2014.

[15] A. Ahangi, M. Karamnejad, N. Mohammadi, R. Ebrahimpour, N. Bagheri, "Multiple classifier system for EEG signal classification with application to brain-computer interfaces", Neural Computing and Applications, 23(5), 1319-1327, 2013.

[16] L. Xiang, Y. Dezhong, D. Wu, L. Chaoyi, "Combining spatial filters for the classification of single-trial EEG in a finger movement task", IEEE Trans. Biomed. Eng, 54, 821-831, 2007.

[17] SM Zhou, JQ Gan, F. Sepulveda, "Classifying mental tasks based on features of higher-order statistics from EEG signals in brain-computer interface", Information Sciences, 178(6):1629-40, 2008.

[18] A.Osman, A. Robert. "Time-course of cortical activation during overt and imagined movements." Proc. Cognitive Neuroscience Annu. Meet., New York 1: 1842-1852, 2001.

[19] Y. Wang, Y.T. Wang, T.P. Jung, "Translation of EEG spatial filters from resting to motor imagery using independent component analysis," PLoS One, vol. 7, no. 5, p. e37665, Jan. 2012.

[20] E. Estrada, H. Nazeran, F. Ebrahimi, M. Mikaeili, "Symmetric Itakura Distance as an EEG Signal Feature for Sleep Depth Determination", American Society of Mechanical Engineers, pp.723-724, 2009.

[21] E. Estrada, P. Nava, H. Nazeran, K. Behbehani, J. Burk, E. Lucas, "Itakura distance: a useful similarity measure between EEG and EOG signals in computer-aided classification of sleep stages", Engineering in Medicine and Biology Society, pp. 1189-1192, 2005.

[22] V. Gonuguntla, Y. Wang, K.C. Veluvolu, "Phase synchrony in subjectspecific reactive band of EEG for classification of motor imagery tasks", InEngineering in Medicine and Biology Society (EMBC), 2784-2787, 2013.

[23] C.J. Stam, G. Nolte, A. Daffertshofer, "Phase lag index: assessment of functional connectivity from multi channel EEG and MEG with diminished bias from common sources", Hum. Brain Mapp., vol. 28, pp. 1178-1193, 2007.

[24] O.D. Eva, A.M. Lazar, "Channels selection for motor imagery paradigm - An Itakura distance based method", E-Health and Bioengineering Conference (EHB), IEEE, pp. 1-4, 2015.

[25] O. D. Eva, D. Tarniceriu, "Substitution of spatial filters from relaxation to motor imagery for EEG based brain computer interface", System Theory, Control and Computing, pp. 147-150, 2015.

[26] O. D. Eva, "Detection and Classification of Mu Rhythm using Phase Synchronization for a Brain Computer Interface" International Journal of Advanced Computer Science and Applications (IJACSA), 7(12), 2016. 
[27] N.F. Ince, A.H. Tewfik, S. Arica, "Extraction subject-specific motor imagery time-frequency patterns for single trial EEG classification", Computers in biology and medicine, 37(4), pp.499-508, 2007.
[28] H. Bashashati, RK Ward, GE Birch, A. Bashashati, "Comparing Different Classifiers in Sensory Motor Brain Computer Interfaces", Yao D, ed. PLOS ONE. 2015;10(6). 